\title{
Stability in a system subject to noise with regulated periodicity
}

\author{
Olga A. Chichigina, ${ }^{1, *}$ Alexander A. Dubkov, ${ }^{2, \dagger}$ Davide Valenti, ${ }^{3, \dagger}$ and Bernardo Spagnolo ${ }^{3, \S}$ \\ ${ }^{1}$ Physics Department, Lomonosov Moscow State University, 119992 Moscow, Russia \\ ${ }^{2}$ Radiophysics Department, Lobachevsky State University of Nizhni Novgorod, 23 Gagarin Avenue, Nizhni Novgorod 603950, Russia \\ ${ }^{3}$ Dipartimento di Fisica, Group of Interdisciplinary Physics and CNISM, Università di Palermo, Viale delle Scienze, I-90128 Palermo, Italy
}

(Received 21 May 2011; published 23 August 2011)

\begin{abstract}
The stability of a simple dynamical system subject to multiplicative one-side pulse noise with hidden periodicity is investigated both analytically and numerically. The stability analysis is based on the exact result for the characteristic functional of the renewal pulse process. The influence of the memory effects on the stability condition is analyzed for two cases: (i) the dead-time-distorted Poissonian process, and (ii) the renewal process with Pareto distribution. We show that, for fixed noise intensity, the system can be stable when the noise is characterized by high periodicity and unstable at low periodicity.
\end{abstract}

DOI: 10.1103/PhysRevE.84.021134

PACS number(s): 05.40.-a, 02.50.-r, 05.10.Gg

\section{INTRODUCTION}

The role played by environmental noise in the dynamics of biological and ecological systems has been a subject of growing interest and investigation in recent years [1-3]. The stochastic nature of living systems and the related noiseinduced effects range from bioinformatics [4,5], to population dynamics [6-9], to virus dynamics and epidemics [10,11].

In a biological context, noise plays a key role in understanding phenomena that can be very difficult to explain by considering deterministic dynamics. Specifically, random variations of species concentrations in natural ecosystems [1,2,11-13], the role of molecular noise in the growth of bacteria that are present in food products [14], and the stochastic dynamics of diseases connected with genetic mutations [15] are fundamental aspects that cannot be neglected when seeking a better understanding of the dynamics of complex living systems. In particular, in these systems the random variations of temperature, food resources, and other environmental parameters can be described using models in which multiplicative noise sources are present $[8,16,17]$. In fact, the models that can effectively describe experimental data in population dynamics are those with multiplicative noise, which can give rise to behavior characterized by a power law $[9,18,19]$.

Multiplicative random fluctuations of natural systems may lead to their instability. This problem, for linear systems with coefficients randomly varying in time, was first studied by mathematicians $[20,21]$ and was investigated further in linear and nonlinear systems by physicists [22-25].

Most of these studies considered Gaussian white or colored noise. However, because of sudden variations in such environmental variables as food resources and, more generally, life conditions, it is also necessary from a biological point of view to model the noise source by a sequence of rectangular pulses with a fixed width and some distribution of probabilities for their heights and times of occurrence. This is done with

\footnotetext{
*chichigina@ilc.edu.ru

†dubkov@rf.unn.ru

$\ddagger$ davide.valenti@unipa.it

${ }^{\S}$ spagnolo@unipa.it
}

the aim of gaining a more realistic and general understanding of the effects of environmental fluctuations. In real systems, because of the presence of pulses, the stochastic perturbations are intrinsically non-Gaussian, and discrete noise models are needed.

The stochastic pulse train is usually modeled by Poissonian white noise (white shot noise) because of the possibility of using the Markovian apparatus of Kolmogorov-Feller equations. This model has already been widely applied in thermal ratchets [26], noise-induced phase transitions [27], and population dynamics [28].

The evolution of the species density in population dynamics, characterized by quasiperiodic cycles with anomalous increases in the population density, has been described by a pulse noise with some delay in Ref. [13]. Moreover, studies on population dynamics show that the competition among species can exhibit quasiperiodic properties and stochastic resonance phenomena $[7,8]$.

A model for epidemic dynamics was analyzed in Refs. [11,29] using a pulse noise model with memory. The pulse noise was the source of the spreading infection, and the memory reflected the presence of the incubation period. The authors found that for a fixed value of the average waiting time of the pulses, more regular infection sources that exhibit some periodicity are less harmful than uncorrelated infection sources.

Measuring the periodicity of the human heartbeat is a crucial topic in medicine. The main focus of the efforts in this direction is related to the super-Poissonian statistics leading to superdiffusion [30-33]. In general, the processes with some kind of periodicity are very popular. In particular, the effects of an external periodic perturbation on a Poissonian rate process and a non-Poissonian rate process have been investigated in Refs. [34] and [35], respectively. Moreover, the role of quasiperiodic perturbations on a Poissonian rate process has been studied in Ref. [36]. This external periodicity or quasiperiodicity can increase or decrease the randomness of the resulting processes.

In this paper, we study the effect of multiplicative noise, in the form of a pulse train with regulated periodicity, on a parametric instability. The specific task of this work is to investigate not only some renewal non-Poissonian processes, but also a regular or periodic process. Moreover, a detailed 
study on the transition from a renewal non-Poissonian process to a periodic process is analyzed. The stability analysis is based on the exact result for the characteristic functional of the renewal point process (see the Appendix). Therefore, the stability problem is solved analytically and the influence of the memory effects on the stability condition for two particular cases is analyzed. A special model of noise source, consisting of a pulse sequence at random times with memory, is presented. The statistical characteristics of the proposed noise model are analyzed. By varying the memory, we can obtain stochastic processes with different characteristics of randomness. The possibility of varying the delay time between pulses allows us to obtain different kinds of correlated noise ranging from white noise, without delay, to a periodic process with delay equal to the average period of the pulse train. We find very good agreement between the theoretical threshold values, characterizing the instability-stability transition, and those obtained by numerical simulations.

The paper is organized as follows. In Sec. II, starting from a discrete model, we construct the equation in continuous time with a multiplicative pulse noise. The exact solution of the stability problem of the considered system is given in Sec. III, where also a comparison with approximate results based on the central limit theorem is presented. Two different cases of a pulse train with regulated periodicity, namely a deadtime-distorted Poissonian process and pulse noise with Pareto distribution of interspike intervals, are analyzed in Sec. IV. The results of numerical simulations are presented in Sec. V, and Sec. VI is devoted to concluding remarks. Finally, in the Appendix we report exact results concerning the characteristic functional of the general renewal pulse process (Poissonian and non-Poissonian).

\section{MODEL}

The pulse noise can therefore be a good candidate to describe the random fluctuations in several biological and physical complex systems. In this section, we present a model of a source of pulse noise that can be used as a multiplicative noise source for a linear system model. The corresponding stochastic linear differential equation is obtained starting from a discrete linear model, sometimes used in the literature [37].

As a noise model, we consider a stochastic process consisting in a random $\delta$-pulse train,

$$
\xi(t)=f \sum_{k} \delta\left(t-t_{k}\right),
$$

where the random time intervals between neighboring $\delta$ pulses $\zeta_{k}=t_{k}-t_{k-1}\left(t_{0}=0\right)$ are mutually independent and identically distributed with a probability density $w(\zeta)$. The process $\xi(t)$ is the so-called renewal process with $\zeta_{k}$ the renewal intervals $[35,38,39]$. Here $f$ is the amplitude of the pulses [40]. The effective (average) period of the random time intervals of the noise defined in Eq. (1) is $T=\langle\zeta\rangle$. Because the noise source has been introduced as a discrete process, the linearized system can be correctly described by the following finite-difference equation:

$$
I_{k+1}=I_{k}\left(1-a \zeta_{k+1}\right)(1+f),
$$

where $I$ is the order parameter of our system model, $a$ is a relaxation parameter, and $0 \leqslant \zeta_{k} \ll 1 / a$ for all $k$. The solution of Eq. (2) reads

$$
I_{k}=I_{0}(1+f)^{k} \prod_{j=1}^{k}\left(1-a \zeta_{j}\right) .
$$

The result given in Eq. (3) can be used to get the approximated solution for a system with continuous time. Therefore, we consider here the conditions for this approximation. Since in the interval $(0, t)$ the number of appearing pulses is

$$
k=\frac{1}{f} \int_{0}^{t} \xi(\tau) d \tau,
$$

for $f \ll 1$ it is easy to transform the term $(1+f)^{k}$ of Eq. (3) into an exponential function by using a well-known remarkable limit. Moreover, representing the product in Eq. (3) as

$$
\prod_{j=1}^{k}\left(1-a \zeta_{j}\right)=\exp \left\{\sum_{j=1}^{k} \ln \left(1-a \zeta_{j}\right)\right\},
$$

and taking into account that $\ln \left(1-a \zeta_{j}\right) \simeq-a \zeta_{j}$ for $a \zeta_{i} \ll 1$, we obtain

$$
I(t) \simeq I_{0} e^{-a t} \exp \left\{\int_{0}^{t} \xi(\tau) d \tau\right\} .
$$

The solution (6) corresponds to the following multiplicative stochastic differential equation

$$
\frac{d I}{d t}=-a I+\xi(t) I
$$

with the initial condition $I(0)=I_{0}$. The noise source is defined according to Eq. (1). The probability distribution and the time characteristics of the noise source $\xi(t)$ play a crucial role in the behavior of such a stochastic system, defined by Eq. (7). Furthermore, we analyze the stability of this system under the following conditions

$$
a T \ll 1, f \ll 1,
$$

where $T=\langle\zeta\rangle$ is the mean waiting time of the next pulse. The conditions (8) follow from our previous considerations. The first one means that the characteristic time of the noise $T$ is much less than the system relaxation time $1 / a$, while the second one implies that the noise is characterized by small values of pulse intensity. However, $\langle\xi\rangle=f / T \sim a$, which indicates that the noise influence cannot be neglected.

\section{STABILITY ANALYSIS}

\section{A. Laplace transform approach}

Starting from Eq. (6), we calculate the $m$-order moment of the order parameter of our system model $I(t)$,

$$
\left\langle I^{m}(t)\right\rangle=I_{0}^{m} e^{-m a t}\left\langle\exp \left\{m \int_{0}^{t} \xi(\tau) d \tau\right\}\right\rangle .
$$

Performing a Laplace transform of Eq. (9) and using Eq. (A19) (see the Appendix), we obtain the following exact result for the Laplace transform of the $m$-order moment

$$
\bar{I}_{m}(s)=I_{0}^{m} \frac{1-\tilde{w}(s+m a)}{(s+m a)\left[1-\tilde{w}(s+m a) e^{m f}\right]},
$$


which is valid under the condition

$$
e^{m f}|\tilde{w}(s+m a)|<1,
$$

and where $\tilde{w}(s)$ is the Laplace transform of the probability density $w(\zeta)$ of random intervals between pulses. Because of the inequality $|\tilde{w}(s+m a)|<\tilde{w}(m a)$, the stability condition of the $m$-order moment of the stochastic process $I(t)$ becomes

$$
f<\frac{1}{m} \ln \frac{1}{\tilde{w}(m a)} .
$$

In particular, for the mean value ( $m=1)$, Eq. (12) reads

$$
f<\ln \frac{1}{\tilde{w}(a)} .
$$

In view of writing the stability condition (12) in a different form, we introduce the critical threshold value $f_{c}$ of the noise amplitude. When $f<f_{c}$, the system is stable; for $f \geqslant f_{c}$, it becomes unstable. The critical value $f_{c}$, defined by inequality (12), is given by

$$
m f_{c}=-\ln \tilde{w}(m a) .
$$

As indicated in Ref. [39], the probability density function $w(\zeta)$ of the waiting times will be either a broad distribution characterized by a power-law fall-off or a narrow distribution with finite moments. In the latter case, the probability that no event occurred up to the time $t$ decays, for $t \rightarrow \infty$, faster than any power law. In the next section, both cases will be considered. Here we restrict our calculations to the case of a narrow distribution. Because of conditions (8), we can retain only the first two terms in the cumulant expansion of the characteristic function $\left\langle e^{i u \zeta}\right\rangle$, obtaining

$$
\ln \left\langle e^{i u \zeta}\right\rangle \simeq i u T-\frac{u^{2} \sigma_{\zeta}^{2}}{2} .
$$

Using Eq. (14), we get

$$
f_{c} \simeq T a-\frac{m a^{2} \sigma_{\zeta}^{2}}{2},
$$

where $\sigma_{\zeta}^{2}$ is the variance of the waiting times. Because of the narrow distribution of waiting times, the second term in Eq. (16) is small. However, we cannot neglect it because the stability of the system depends very sensitively on the critical value. The same system can be stable in the presence of exactly periodic pulses ( $\sigma_{\zeta}^{2}=0$, i.e., absence of noise) and unstable for completely random pulses $\left(\sigma_{\zeta}^{2} \sim T^{2}\right.$, i.e., uncorrelated noise). In other words, for fixed relaxation parameter $a$, the critical value of the noise amplitude $f_{c}$ at high periodicity is higher than that obtained at low periodicity.

The expression (16) is in agrement with the stability condition obtained in Ref. [25]. There, the stability condition, expressed through high-order spectral densities of the noise signal, turns out to be the same as that of this work when the truncated expansion (15), valid for narrow distribution, is used. The general idea that the stability is related to the variance of the noise fluctuations was given in Ref. [41].
From Eq. (16) we obtain, for fixed noise amplitude, the expression of the critical value of the relaxation parameter $a_{c}$ as a function of the other system parameters,

$$
a_{c} \simeq \frac{f}{T}+\frac{m f^{2} \sigma_{\zeta}^{2}}{2 T^{3}} .
$$

\section{B. Central limit theorem approach}

In asymptotics $(t \rightarrow \infty)$, the integral of the renewal pulse noise (1),

$$
\alpha(t)=\int_{0}^{t} \xi(\tau) d \tau,
$$

can be represented as the sum of a very large number of independent identically distributed random variables. According to the central limit theorem (CLT), in this limit, the probability distribution of the random process $\alpha(t)$ tends to a Gaussian. As a consequence for large $t$, from Eq. (9) we have

$$
\begin{aligned}
\left\langle I^{m}(t)\right\rangle & =I_{0}^{m} e^{-m a t}\left\langle e^{m \alpha(t)}\right\rangle \\
& \simeq I_{0}^{m} \exp \left\{m\langle\alpha(t)\rangle+\frac{m^{2}}{2} \sigma_{\alpha}^{2}(t)-m a t\right\} .
\end{aligned}
$$

Now we have to calculate the mean value $\langle\alpha(t)\rangle$ and the variance $\sigma_{\alpha}^{2}(t)$. Using the same procedure as that used in the Appendix, we obtain

$$
\begin{aligned}
\langle\alpha(t)\rangle & =\left\langle\int_{0}^{t} \xi(\tau) d \tau\right\rangle=f \sum_{n=1}^{\infty} n P_{n}(t) \\
& =f\langle n(t)\rangle \simeq \frac{f}{T} t+\frac{f}{2}\left(\frac{\sigma_{\zeta}^{2}}{T^{2}}-1\right),
\end{aligned}
$$

where $n(t)$ is the random number of pulses in the interval $(0, t)$. Analogously, the second moment and variance are obtained,

$$
\begin{aligned}
\left\langle\alpha^{2}(t)\right\rangle & =\left\langle\left[\int_{0}^{t} \xi(\tau) d \tau\right]^{2}\right\rangle=f^{2} \sum_{n=1}^{\infty} n^{2} P_{n}(t) \\
& =f^{2}\left\langle n^{2}(t)\right\rangle \simeq \frac{f^{2}}{T^{2}} t^{2}+\frac{f^{2}}{T}\left(\frac{2 \sigma_{\zeta}^{2}}{T^{2}}-1\right) t, \\
\sigma_{\alpha}^{2}(t) & =\left\langle\alpha^{2}(t)\right\rangle-\langle\alpha(t)\rangle^{2} \simeq \frac{f^{2} \sigma_{\zeta}^{2}}{T^{3}} t .
\end{aligned}
$$

Formulas (20) and (21) were obtained in Ref. [38] using the probability-generating function and calculating the Laplace transforms of $\langle n(t)\rangle$ and $\left\langle n^{2}(t)\right\rangle$. Substitution of Eqs. (20) and (21) in Eq. (19) gives in asymptotics $(t \rightarrow \infty)$

$$
\left\langle I^{m}(t)\right\rangle \simeq I_{0}^{m} \exp \left\{m\left(\frac{f}{T}+\frac{m f^{2} \sigma_{\zeta}^{2}}{2 T^{3}}-a\right) t\right\} .
$$

Equation (22) provides the same critical value of the relaxation parameter as that given in Eq. (17), obtained by renewal theory and the Laplace transform method. This indicates that in the framework of our approximation, the two methods provide the same results. 


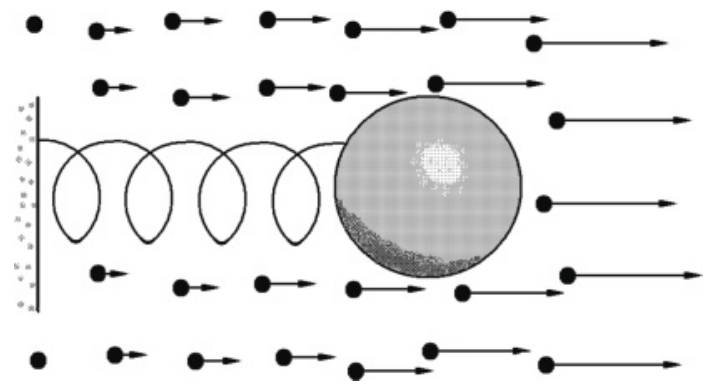

FIG. 1. The mechanical model of the simplest process with quasiperiodic multiplicative noise. A large particle moves in a parabolic potential under the influence of a directed flow of small accelerating particles. The system is overdamped. The flow of particles is quasiperiodic and can be considered as statistically asymmetrical noise. The stability of the system depends not only on the intensity of the flow but also on its periodicity.

\section{NOISE WITH HIDDEN PERIODICITY}

\section{A. Statistical characteristics}

\section{Dead-time-distorted Poissonian process}

From a physical point of view, the order parameter $I(t)$ can describe the coordinate of a light particle under the influence of a regular force (characterized by the parameter $a$ ) acting in one direction and a random flow of particles acting in the opposite direction (see Fig. 1). In this case, $f$ represents the forces due to the collisions among particles.

Now we analyze the stochastic process (1) with the following probability distribution of intervals between pulses:

$$
w(\zeta)= \begin{cases}0, & \zeta<\zeta_{0} \\ \lambda e^{-\lambda\left(\zeta-\zeta_{0}\right)}, & \zeta>\zeta_{0}\end{cases}
$$

where $\lambda$ and $\zeta_{0}$ are positive real numbers. Here $\zeta_{0}$ is responsible for the randomness or periodicity of the flow (see Fig. 1). As is evident from Eq. (23), within the time interval $\zeta_{0}$, after each $\delta$ pulse, the occurrence of a new pulse is forbidden. The stochastic process (1) with the probability distribution $w(\zeta)$ of time intervals in the form of Eq. (23) is known as a dead-time-distorted Poissonian process and can be considered as an example of a renewal pulse process. Similar dead-timedistorted Poissonian processes are presented in Refs. [42,43] as renewal pulse processes in quantum optics. In Ref. [44], the presence of periodic properties of the process is shown by means of experiments where radiation from a ${ }^{22} \mathrm{Na}$ source is used.

As is seen from Eq. (23), in the limit $\lambda \rightarrow \infty$ for fixed $\zeta_{0}$ we find pure deterministic periodic excitations with time intervals between pulses characterized by the probability distribution $w(\zeta)=\delta\left(\zeta-\zeta_{0}\right)$. In the opposite situation, i.e., $\zeta_{0}=0$, the stochastic process $\xi(t)$ becomes the white Poissonian noise. Thus, the parameter of periodicity $\zeta_{0} / T$ ranges from 0 to 1 .

The rate of pulse train $\lambda$ can be expressed in terms of the mean waiting time $T=\langle\zeta\rangle$,

$$
\langle\zeta\rangle=\zeta_{0}+\frac{1}{\lambda}
$$

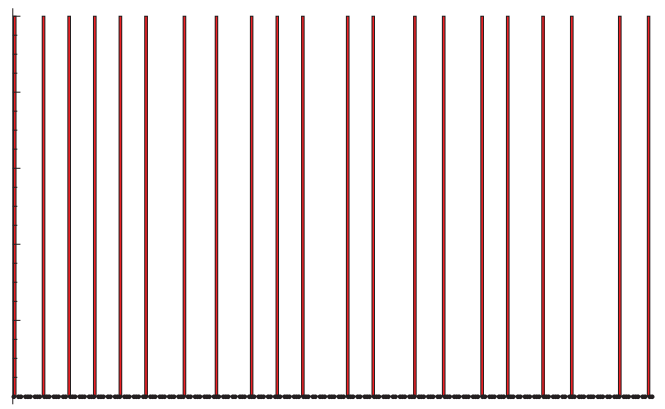

FIG. 2. (Color online) The pulse train presenting peaks with parameter of periodicity $\zeta_{0} / T$ equal to 0.8 (high periodicity). The period $T$ is equal to $100 \mathrm{~s}$. There is some delay $\zeta_{0}=80 \mathrm{~s}$ (some time interval) after each pulse during which the next pulse is impossible. After this delay, the rate $\lambda$ of the pulse train is equal to 0.05 .

For a fixed effective period $T$, the rate $\lambda$ increases as the memory $\zeta_{0}$ increases (see Figs. 2 and 3). In particular, $\lambda \rightarrow \infty$ when $\zeta_{0} \rightarrow T$. Because of the simple "structure" of this noise, it is possible to calculate all its characteristics. The standard deviation $\sigma_{\zeta}^{2}$ of the time distances is

$$
\sigma_{\zeta}^{2}=\left\langle\zeta^{2}\right\rangle-\langle\zeta\rangle^{2}=\left(T-\zeta_{0}\right)^{2}
$$

From Eq. (23), we can get the probability distribution for the occurrence of the $n$th pulse,

$$
W_{t_{n}}(\tau)=\frac{\lambda^{n}\left(\tau-n \zeta_{0}\right)^{n-1}}{(n-1) !} e^{-\lambda\left(\tau-n \zeta_{0}\right)} 1\left(\tau-n \zeta_{0}\right),
$$

where $1(x)$ is the step function. Throughout this paper, we suppose that at time $t_{0}=0$ there is a pulse so that $W_{t_{n}}(\tau)=W_{t_{n}}\left(\tau \mid t_{0}\right)$ [13]. The distributions are shown in Fig. 4 for $n=2,3$ and two different values of periodicity, namely $\zeta_{0} / T=0.35,0.85$. From Eq. (26), we get, for the $n$th pulse, the average time $\left\langle t_{n}\right\rangle=n T$ and the most probable time $\tau_{n}^{\max }=(n-1) T+\zeta_{0}$. The probability distribution at $\tau_{n}^{\max }$ is

$$
W_{t_{n}}\left(\tau_{n}^{\max }\right)=\max W_{t_{n}}=\frac{\lambda(n-1)^{n-1} e^{-(n-1)}}{(n-1) !} .
$$

We can also calculate the variance

$$
\left\langle\left(\Delta t_{n}\right)^{2}\right\rangle=n\left(T-\zeta_{0}\right)^{2},
$$

which is zero in the periodic case.

The correlation function of the renewal pulse noise $\xi(t)$,

$$
K(\tau)=\left\langle\xi \xi_{\tau}\right\rangle-\langle\xi\rangle^{2}=\left\langle\xi \xi_{\tau}\right\rangle-\frac{f^{2}}{T^{2}},
$$

is obtained by the formula connecting the characteristic function of random intervals between pulses $\Theta(i s)$ and the Laplace transform $L[K(\tau) ; s]$ of the correlation function $K(\tau)$ [see Eq. (6.99) in Ref. [40]],

$$
L[K(\tau) ; s]=\frac{f^{2}}{T}\left[\frac{1+\Theta(i s)}{2[1-\Theta(i s)]}-\frac{1}{s T}\right] .
$$

Because of the relation

$$
\Theta(i s)=\left\langle e^{i(i s) \zeta}\right\rangle=\int_{0}^{\infty} e^{-s \zeta} w(\zeta) d \zeta=\tilde{w}(s),
$$




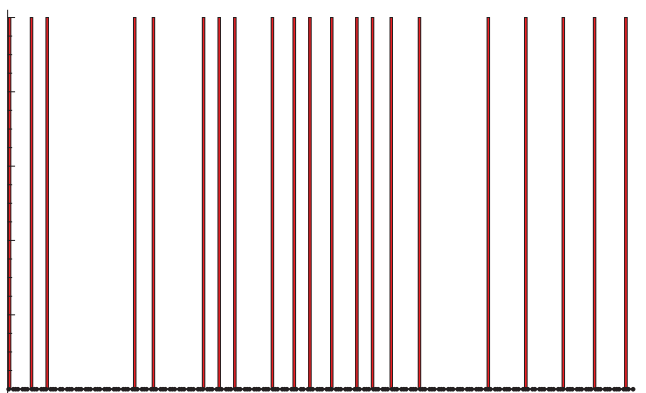

FIG. 3. (Color online) The pulse train presenting peaks with parameter of periodicity $\zeta_{0} / T$ equal to 0.4 (low periodicity). Period $T$ is equal to $100 \mathrm{~s}$. There is some delay $\zeta_{0}=40 \mathrm{~s}$ (some time interval) after each pulse during which the next pulse is impossible. After this delay, the rate $\lambda$ of the pulse train is equal to 0.017 .

$\Theta(i s)$ is the Laplace transform of the probability distribution $w(\zeta)$. By series expansion of the factor $1 /[1-\tilde{w}(s)]$, from Eqs. (30) and (31) we get

$$
L[K(\tau) ; s]=\frac{f^{2}}{T}\left\{\frac{1}{2}+\sum_{n=1}^{\infty} \tilde{w}^{n}(s)-\frac{1}{s T}\right\} .
$$

The inverse Laplace transform of this equation gives finally the correlation function of the renewal pulse noise (1),

$$
K(\tau)=\frac{f^{2}}{T}\left[\delta(\tau)+\sum_{n=1}^{\infty} W_{t_{n}}(|\tau|)-\frac{1}{T}\right],
$$

where the Dirac $\delta$ function corresponds to the correlation of the pulse with itself. In the case of white Poissonian noise, the second and third terms cancel each other and we have a $\delta$-correlated noise. The behavior of the correlation function $K(\tau)$ [Eq. (33)] versus the time is shown in Fig. 5 for two values of the ratio $\zeta_{0} / T$, namely $\zeta_{0} / T=0.35,0.85$. We see that in the case of long memory $\left(\zeta_{0} / T=0.85\right), K(\tau)$ is quasiperiodic, and for short memory (or small periodicity) $\left(\zeta_{0} / T=0.35\right)$, it goes quickly to zero.

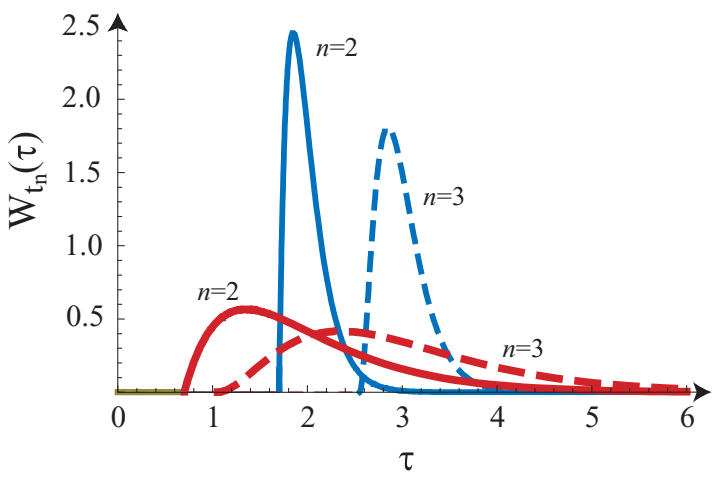

FIG. 4. (Color online) The probability distributions of the appearance times for the second (solid blue line, with the highest peak) and third (dashed blue line, with the second highest peak) pulses in the process with $\zeta_{0} / T=0.85$ and for the second (solid red line, with the third highest peak) and third (dashed red line, with the lowest peak) pulses in the process with $\zeta_{0} / T=0.35$. The effective period is $T=1$. The lower the periodicity or the higher the number of pulse, the wider and more Gaussian-like is the probability distribution.

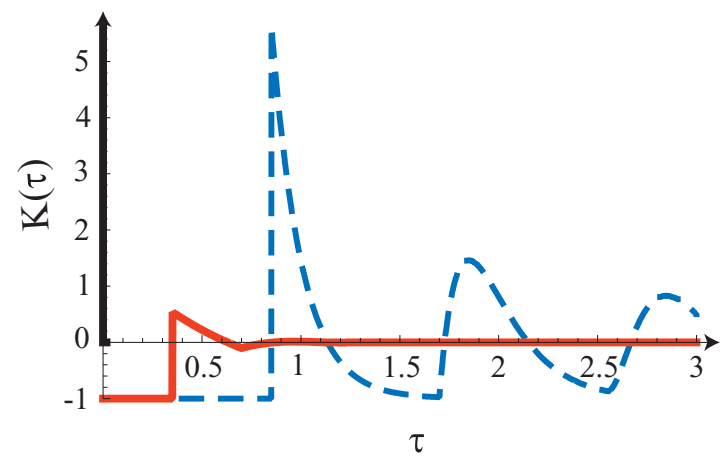

FIG. 5. (Color online) The time behavior of the correlation function $K(\tau)$ for the dead-time-distorted Poissonian process with $\zeta_{0} / T=0.35$ (solid red line) and for the process with $\zeta_{0} / T=0.85$ (dashed blue line). The effective period is $T=1$. In the case of low periodicity, only the first pulses are correlated. The vertical black tick line at $\tau=0$ represents the $\delta$ function of Eq. (33).

Since the correlation function is nonmonotonic, we cannot use the equivalent rectangular method to estimate the correlation time $\tau_{\text {cor }}$. However, it can be estimated by the ratio of the correlation function calculated at the first maximum, $K\left(\tau_{1}^{\max }\right)$, over the absolute value of the same correlation function calculated close to zero, $\left|K\left(0^{+}\right)\right|=f^{2} / T^{2}$. In this way, we obtain

$$
\frac{\tau_{\text {cor }}}{T}=\frac{K\left(\tau_{1}^{\max }\right)}{\left|K\left(0^{+}\right)\right|} .
$$

Substituting Eq. (27), calculated in the limit $n \rightarrow 1$ in Eq. (33) and then in Eq. (34), we have

$$
\tau_{\mathrm{cor}}=\frac{T \zeta_{0}}{T-\zeta_{0}}
$$

As one can see from Eq. (35), the correlation time goes to zero for white Poissonian noise $\left(\zeta_{0}=0\right)$, and takes on very large values in the presence of a periodic process $\left(\zeta_{0} \rightarrow T\right)$. For the correlation function shown in Fig. 5, the values of $\tau_{\text {cor }}$ are equal to 5.67 (dashed blue line) and 0.54 (solid red line), which is in good agreement with the visual estimation.

The standard deviation of the number of pulses at the time $t$ can be estimated by the standard deviation of the $n$th pulse, where $n=t / T$. For one realization of $n$ pulses, the event in which the $n$th pulse occurred at the time $t=t_{n}+\Delta t_{n}$ corresponds to the event in which the number of pulses at the moment $t_{n}$ is about $n-\Delta t_{n} / T$. This implies

$$
\Delta n \approx-\frac{\Delta t_{n}}{T}
$$

The second moment of this value reads

$$
\sigma_{n}^{2}(t)=\left\langle(\Delta n)^{2}\right\rangle \approx \frac{\left\langle\left(\Delta t_{n}\right)^{2}\right\rangle}{T^{2}}=\frac{\left(T-\zeta_{0}\right)^{2}}{T^{3}} t .
$$

Substituting the standard deviation $\sigma_{\zeta}^{2}$ of Eq. (25) in the second equation of Eqs. (21) and dividing by $f^{2}$, we arrive at the same result of Eq. (37). 


\section{Pulse train with Pareto distribution}

Another model of noise with hidden periodicity is based on the renewal process with Pareto law for the probability density function of the waiting times,

$$
w(\zeta)= \begin{cases}0, & \zeta<\zeta_{0} \\ \mu \zeta_{0}^{\mu} / \zeta^{1+\mu}, & \zeta>\zeta_{0}\end{cases}
$$

where $\mu$ and $\zeta_{0}$ are positive real numbers. The power-law probability distribution (38) occurs in many social, economic, geophysical, biological, and physical systems and has significant consequences for our understanding of natural phenomena $[45,46]$. We note that the distribution (38), for $\mu>1$, has a finite first-order moment, which reads

$$
T=\langle\zeta\rangle=\frac{\mu \zeta_{0}}{\mu-1}
$$

According to Eq. (39), the parameter of periodicity $\zeta_{0} / T$ is equal to $1-1 / \mu$ and ranges from 0 to 1 with $\mu \in(1, \infty)$. The variance of the time distances between pulses is

$$
\sigma_{\zeta}^{2}=\frac{T^{2}}{\mu(\mu-2)},
$$

and it is valid for $\mu>2$. As follows from Eqs. (39) and (40), in the limit $\mu \rightarrow \infty$ for a fixed value of $\zeta_{0}$ we obtain pure deterministic periodic excitations with probability distribution of the time intervals between pulses given by $w(\zeta)=\delta\left(\zeta-\zeta_{0}\right)$. To find the spectral power density of the stochastic process $\xi(t)$ given in Eq. (1), we can use the well-known result [40]

$$
S_{\xi-\langle\xi\rangle}(\omega)=\frac{2 f^{2}}{T} \operatorname{Re}\left\{\frac{1+\tilde{w}(i \omega)}{1-\tilde{w}(i \omega)}\right\} .
$$

Here $\tilde{w}(s)$ is the Laplace transform of the Pareto distribution (38),

$$
\tilde{w}(s)=\mu\left(s \zeta_{0}\right)^{\mu} \Gamma\left(-\mu, s \zeta_{0}\right),
$$

where $\Gamma(\alpha, z)$ is the incomplete Gamma function,

$$
\Gamma(\alpha, z)=\int_{z}^{\infty} t^{\alpha-1} e^{-t} d t .
$$

\section{B. Stability condition in the case of noise with regulated periodicity}

Starting from the probability distribution of Eq. (23), after some algebraic manipulations, we find its Laplace transform,

$$
\tilde{w}(s)=\frac{\lambda e^{-s \zeta_{0}}}{\lambda+s} .
$$

Afterward, by using Eq. (44) in the stability condition (12), we get

$$
f<a \zeta_{0}+\frac{1}{m} \ln \left[1+m a\left(T-\zeta_{0}\right)\right] .
$$

The corresponding expression for the critical value of the noise amplitude is

$$
f_{c}=a \zeta_{0}+\frac{1}{m} \ln \left[1+m a\left(T-\zeta_{0}\right)\right] .
$$

In the case of white Poissonian noise $\left(\zeta_{0}=0\right)$, inequality (45) becomes

$$
f<\frac{\ln (1+m a T)}{m} .
$$

Conversely, for periodic pulse excitation, i.e., $\zeta_{0}=T$ or, equivalently, $\lambda \rightarrow \infty$, inequality (45) reduces to $f<a T$.

Because of the general conditions (8), from inequality (45) we obtain the following approximate critical value for the noise amplitude

$$
f_{c} \simeq a T-\frac{m a^{2} \sigma_{\zeta}^{2}}{2}
$$

which coincides with that of Eq. (16). This value corresponds to the stability-instability transition of the $m$ th moment of the system described by Eq. (7). When the noise source has some periodicity, this system shows qualitatively different behaviors depending on the values of the noise amplitude and periodicity.

\section{Stability condition in the case of noise with Pareto distribution of interspike intervals}

For a $\delta$-pulse train with Pareto distribution of the time intervals, in accordance with Eq. (42), the stability condition (12) takes the form

$$
f<\frac{1}{m} \ln \left[\frac{1}{\mu\left(m a \zeta_{0}\right)^{\mu} \Gamma\left(-\mu, m a \zeta_{0}\right)}\right] .
$$

Because of the conditions (8) and Eq. (39), we have $a \zeta_{0} \ll 1$. Using this condition, together with the following $\mu>2$, by the power-series expansion of the incomplete Gamma function (43),

$$
\Gamma(\alpha, z)=\Gamma(\alpha)-\sum_{n=0}^{\infty} \frac{(-1)^{n} z^{\alpha+n}}{n !(\alpha+n)},
$$

we can obtain a simpler expression for the stability condition. Here $\Gamma(\alpha)$ is the Gamma function. We have

$$
\begin{aligned}
f & <-\frac{1}{m} \ln \left[\mu\left(m a \zeta_{0}\right)^{\mu} \Gamma(-\mu)-\sum_{n=0}^{\infty} \frac{(-1)^{n}\left(m a \zeta_{0}\right)^{n} \mu}{n !(n-\mu)}\right] \\
& \simeq-\frac{1}{m} \ln \left[1-\left(m a T-\frac{1}{2}(\mu-1)^{2}\left(m a \sigma_{\zeta}\right)^{2}\right)\right]
\end{aligned}
$$

By using the truncated series expansion

$$
-\ln (1-x) \simeq x+\frac{x^{2}}{2},
$$

we get

$$
f<-\frac{1}{m}\left[m a T-\frac{1}{2}(\mu-1)^{2}\left(m a \sigma_{\zeta}\right)^{2}+\frac{(m a T)^{2}}{2}\right],
$$

which, after simple algebra, gives the final expression for the stability condition,

$$
f<a T\left(1-\frac{m a \sigma_{\zeta}^{2}}{2 T}\right)
$$

The critical value of the noise amplitude for this pulse process with Pareto distribution is then

$$
f_{c} \simeq T a-\frac{m a^{2} \sigma_{\zeta}^{2}}{2}
$$

which coincides with that of Eq. (16). 


\section{NUMERICAL SIMULATIONS}

In this section, we show the results obtained by solving numerically Eq. (7), and we compare them with the analytical findings obtained in Sec. IV for the dead-time-distorted Poissonian process. The numerical solution of Eq. (7) was calculated by setting $I(0)=0.5$ (initial value), $a=2$ (relaxation parameter), and $T=10 \tau$ (effective period). The integration step, i.e., the pulse width $\tau$, is $\Delta t=10^{-4}$. After calculating the time series of $I(t)$, we obtained the time behavior of the second moment, $\left\langle I^{2}(t)\right\rangle$, averaging over an ensemble of 100 trajectories. In particular, the second moment was calculated for different values of the memory $\zeta_{0}$, i.e., the delay before a new pulse can occur.

In Figs. 6 and 7, we show the time behavior of the second moment, $\left\langle I^{2}(t)\right\rangle$, for three different values of the noise amplitude $f$, namely $f=f_{c}, f_{c} \pm \varepsilon$ with $f_{c}=1.996 \times$ $10^{3}, 1.999 \times 10^{3}, \varepsilon=2 \times 10^{-5}$, and two values of the scaled memory parameter $\zeta_{0} / T=0,0.6$. In the two figures, we used the critical value $f_{c}$ given by Eq. (46) [whose approximated expression coincides with Eq. (16)], obtaining the average curves of $\left\langle I^{2}(t)\right\rangle$ for $f=f_{c}$ (red dotted line), $f>f_{c}$ (blue dashed line), and $f<f_{c}$ (black solid line). Both the results in Fig. 6 (absence of memory $\rightarrow \zeta_{0} / T=0$ ) and Fig. 7 (intermediate value of memory $\rightarrow \zeta_{0} / T=0.6$ ) indicate that the time behavior of $\left\langle I^{2}(t)\right\rangle$ is qualitatively different depending on the values of the noise amplitude. In particular, the results show that $\left\langle I^{2}(t)\right\rangle$ is constant for $f=f_{c}$, increases for $f>f_{c}$, and decreases for $f<f_{c}$. This indicates that, varying the value of $f$ below and above $f_{c}$, a stability-instability transition occurs, what means that the noise amplitude $f$ has a critical value that coincides with the value $f_{c}$ obtained analytically [see formula (46) for $m=2$ ]. Moreover, we compared numerical and analytical results obtained for other values of the memory $\zeta_{0}$, and we found perfect agreement.

We also checked our numerical approach for other moments. Specifically, we calculated $\langle I(t)\rangle$ and $\left\langle I^{m}(t)\right\rangle$ with $m=$

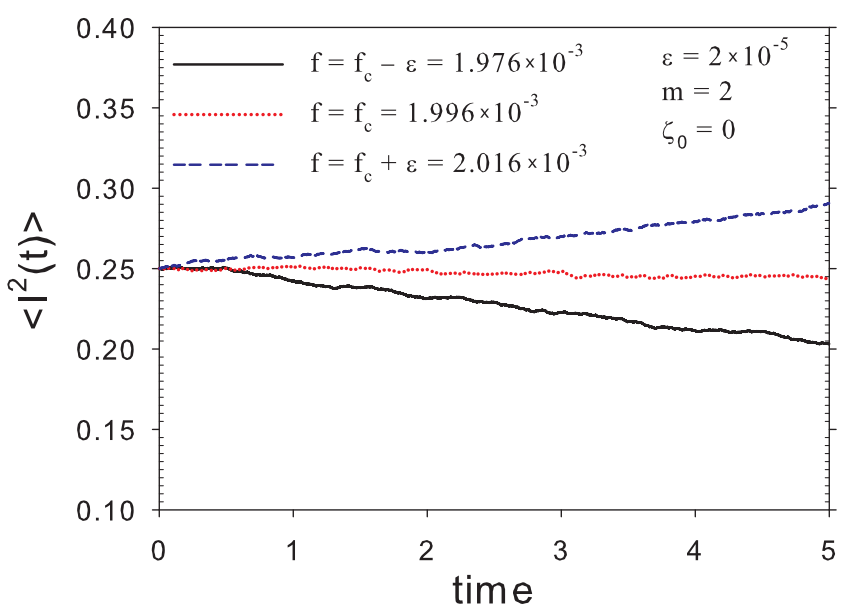

FIG. 6. (Color online) The time behavior of the second moment, $\left\langle I^{2}(t)\right\rangle$, for three different values of the noise amplitude $f$, namely $f=f_{c}, f_{c} \pm \varepsilon$ with $f_{c}=1.996 \times 10^{3}, \varepsilon=2 \times 10^{-5}$. The value of the scaled memory parameter is $\zeta_{0} / T=0$ (absence of memory), corresponding to a white Poissonian noise source.

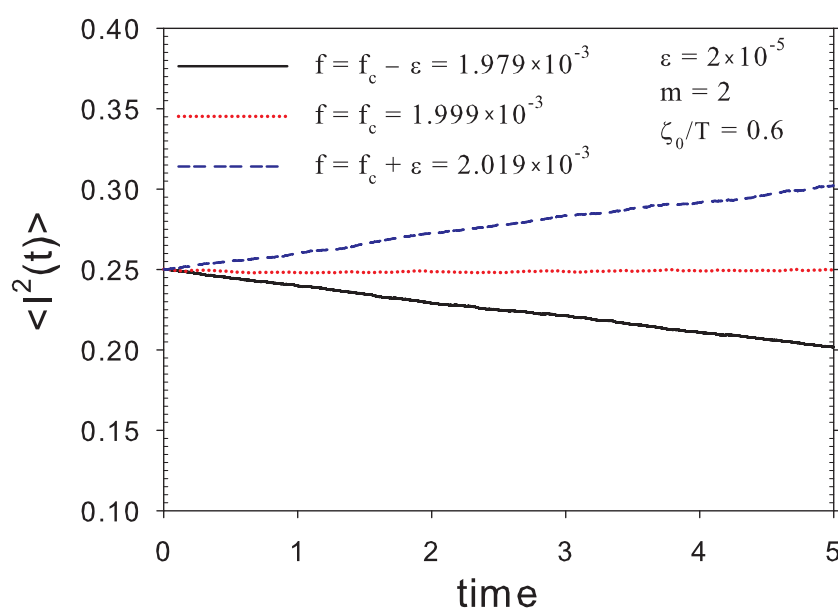

FIG. 7. (Color online) The time behavior of the second moment, $\left\langle I^{2}(t)\right\rangle$, for three different values of the noise amplitude $f$, namely $f=f_{c}, f_{c} \pm \varepsilon$ with $f_{c}=1.999 \times 10^{3}, \varepsilon=2 \times 10^{-5}$. The value of the scaled memory parameter is $\zeta_{0} / T=0.6$, corresponding to a deadtime distorted Poissonian process.

$3, \ldots, 10$. The results, not shown here, indicate that the critical values obtained from Eq. (46) represent the threshold values for the stability-instability transition also for these moments. Moreover, we observe that, according to Eq. (46), the values of $f_{c}$ increase as the memory $\zeta_{0}$ increases. This means that, during the time evolution of the system, the uncorrelated pulse noise is responsible, with respect to the pulse noise with some periodicity, for a larger increase of the generic moment $\left\langle I^{m}(t)\right\rangle$. Our analysis allows us to conclude that, for fixed values of the relaxation parameter and noise amplitude, a larger delay $\zeta_{0}$ (more correlated noise) determines a greater stability of the system.

\section{CONCLUSIONS}

The stability problem for systems under the influence of quasiperiodic pulse noise is solved analytically. A special noise model, consisting of a pulse sequence with random times and memory, is presented. This noise source affects the dynamics of the order parameter process $I(t)$, which is also subject to a deterministic force whose value depends on a relaxation parameter $a$. By varying the values of the memory, i.e., delay time between two successive pulses, we obtain a process with different levels of randomness. Specifically, we find that varying the values of the delay time produces different kinds of correlated noise ranging from white Poissonian noise to a periodic process with delay equal to the average period of the pulse train. When the noise with periodicity is included in the equation, we observe qualitatively different behavior of the system depending on the values of the noise amplitude and periodicity. In particular, for each moment $\left\langle I^{m}(t)\right\rangle$ we calculate a critical value $f_{c}$ of the noise amplitude. Below and above this critical value, the generic moment $\left\langle I^{m}(t)\right\rangle$ shows stable and unstable time behavior, respectively, undergoing a stability-instability transition. Two different cases of pulse train with regulated periodicity, namely a dead-time-distorted 
Poissonian process and pulse noise with Pareto distribution of interspike intervals, are analyzed.

An exact analytical expression of the characteristic functional of the general renewal pulse process (Poissonian and non-Poissonian) is derived in the Appendix. Moreover, the characteristic function for two particular cases is derived, namely for a Poissonian pulse noise source and for the continuous time random walk (CTRW) process.

By numerically solving the stochastic differential equation for $I(t)$, in the case of the dead-time-distorted Poissonian process, we calculate again the time behavior of the moments $\left\langle I^{m}(t)\right\rangle$ and the corresponding critical values of the noise amplitude $f$. The results obtained are in perfect agreement with the analytical findings.

Finally, we note that a great variety of natural phenomena, such as nuclear decay, autocatalytic chemical reactions, transient dynamics of pulse lasers, spread of epidemics, and population dynamics, show characteristics of randomness that can be well modeled by a source of correlated pulse noise, such as that presented in this work.

\section{ACKNOWLEDGMENTS}

The authors are grateful to Igor M. Sokolov for useful discussions. We also acknowledge the support of the Rus- sian Foundation for Basic Research (project 11-02-01418) and Ministero dell'Istruzione, dell'Università e della Ricerca (Italian Government).

\section{APPENDIX}

Here we consider a noise source consisting of $\delta$-pulse trains more general than that given in Eq. (1),

$$
\xi(t)=\sum_{k} a_{k} \delta\left(t-t_{k}\right),
$$

with the random amplitudes of the pulses $a_{k}$, which are assumed to be statistically independent from each other and identically distributed with the probability density function $W_{a}(x)$. The random process (A1) represents the first derivative of the well-known CTRW model [47] and the amplitudes $a_{k}$ are the values of random jumps. Furthermore, we will find its characteristic functional, which is equal, by definition, to the following average

$$
\Theta_{t}[u]=\left\langle\exp \left\{i \int_{0}^{t} \xi(\tau) u(\tau) d \tau\right\}\right\rangle,
$$

where $u(t)$ is an arbitrary deterministic function. Substituting Eq. (A1) in Eq. (A2), using the theorem of total probability and the property of the $\delta$ function, we arrive at

$$
\Theta_{t}[u]=\left\langle\exp \left\{i \int_{0}^{t} \sum_{k} a_{k} \delta\left(\tau-t_{k}\right) u(\tau) d \tau\right\}\right\rangle_{a_{k}, t_{k}}=\left\langle 1\left(t_{1}-t\right)\right\rangle+\sum_{n=1}^{\infty}\left\langle 1\left(t-t_{n}\right) 1\left(t_{n+1}-t\right) \prod_{k=1}^{n} e^{i a_{k} u\left(t_{k}\right)}\right\rangle_{a_{k}, t_{k}}
$$

or because of statistically independent and identically distributed amplitudes $a_{k}$,

$$
\Theta_{t}[u]=\left\langle 1\left(t_{1}-t\right)\right\rangle+\sum_{n=1}^{\infty}\left\langle 1\left(t-t_{n}\right) 1\left(t_{n+1}-t\right) \prod_{k=1}^{n} \theta_{a}\left(u\left(t_{k}\right)\right)\right\rangle_{t_{k}},
$$

where $\theta_{a}(u)=\left\langle e^{i u a}\right\rangle$ is the characteristic function of the amplitudes.

By averaging over the times of the pulse appearance $t_{k}$ and taking into account that $t_{1}<t_{2}<\cdots<t_{n+1}$, Eq. (A3) becomes

$$
\Theta_{t}[u]=\int_{t}^{\infty} W_{t_{1}}(\tau) d \tau+\sum_{n=1}^{\infty} \int_{t}^{\infty} d \tau_{n+1} \int_{0}^{t} \theta_{a}\left(u\left(\tau_{n}\right)\right) d \tau_{n} \ldots \int_{0}^{\tau_{2}} \theta_{a}\left(u\left(\tau_{1}\right)\right) W_{t_{1} \cdots t_{n+1}}\left(\tau_{1}, \ldots, \tau_{n+1}\right) d \tau_{1},
$$

where $W_{t_{1} \cdots t_{n}}\left(\tau_{1}, \ldots, \tau_{n}\right)$ is the joint probability distribution of the random times of the pulses' appearance. Because $t_{n+1}=$ $t_{n}+\zeta_{n+1}$, we can use the formula for the conditional probability density function and take into account the statistical independence of the time intervals between neighboring pulses $\zeta_{k}$. As a result, we find

$$
W_{t_{1} \cdots t_{n+1}}\left(\tau_{1}, \ldots, \tau_{n+1}\right)=W_{t_{1} \cdots t_{n}}\left(\tau_{1}, \ldots, \tau_{n}\right) W_{t_{n+1}}\left(\tau_{n+1} \mid \tau_{1}, \ldots, \tau_{n}\right)=W_{t_{1} \cdots t_{n}}\left(\tau_{1}, \ldots, \tau_{n}\right) w\left(\tau_{n+1}-\tau_{n}\right),
$$

and hence

$$
W_{t_{1} \cdots t_{n+1}}\left(\tau_{1}, \ldots, \tau_{n+1}\right)=w\left(\tau_{1}\right) w\left(\tau_{2}-\tau_{1}\right) \times \cdots \times w\left(\tau_{n+1}-\tau_{n}\right) .
$$

After substituting Eq. (A5) into Eq. (A4), we obtain

$$
\Theta_{t}[u]=\int_{t}^{\infty} w(\tau) d \tau+\sum_{n=1}^{\infty} \int_{t}^{\infty} d \tau \int_{0}^{t} \theta_{a}\left(u\left(\tau_{n}\right)\right) w\left(\tau-\tau_{n}\right) d \tau_{n} \cdots \int_{0}^{\tau_{2}} \theta_{a}\left(u\left(\tau_{1}\right)\right) w\left(\tau_{2}-\tau_{1}\right) w\left(\tau_{1}\right) d \tau_{1}
$$

or

$$
\Theta_{t}[u]=\int_{t}^{\infty} d \tau\left(w(\tau)+\sum_{n=1}^{\infty} \int_{0}^{t} \theta_{a}\left(u\left(\tau_{1}\right)\right) w\left(\tau-\tau_{1}\right) d \tau_{1} \cdots \int_{0}^{\tau_{n-1}} \theta_{a}\left(u\left(\tau_{n}\right)\right) w\left(\tau_{n-1}-\tau_{n}\right) w\left(\tau_{n}\right) d \tau_{n}\right) .
$$


The series on the right-hand side of Eq. (A6) represents the result of some iterative procedure and can be written in the more compact form,

$$
\begin{aligned}
\Theta_{t}[u]= & \int_{t}^{\infty} d \tau \int_{0}^{t} \theta_{a}\left(u\left(\tau_{1}\right)\right) w\left(\tau-\tau_{1}\right) R_{\tau_{1}}[u] d \tau_{1} \\
& +\int_{t}^{\infty} w(\tau) d \tau
\end{aligned}
$$

where the auxiliary functional $R_{t}[u]$ should be found from the following integral equation

$$
R_{t}[u]=w(t)+\int_{0}^{t} \theta_{a}(u(\tau)) w(t-\tau) R_{\tau}[u] d \tau .
$$

Due to Eq. (A8) one can rewrite Eq. (A7) in the form

$$
\begin{aligned}
\Theta_{t}[u]= & -\int_{t}^{\infty} d \tau \int_{t}^{\tau} \theta_{a}\left(u\left(\tau_{1}\right)\right) w\left(\tau-\tau_{1}\right) R_{\tau_{1}}[u] d \tau_{1} \\
& +\int_{t}^{\infty} R_{\tau}[u] d \tau .
\end{aligned}
$$

After taking the time derivative, we obtain

$$
\dot{\Theta}_{t}[u]=\left[\theta_{a}(u(t))-1\right] R_{t}[u] .
$$

After integrating Eq. (A9) with the initial condition $\Theta_{0}[u]=$ 1, which follows from Eq. (A2), we finally get

$$
\Theta_{t}[u]=1+\int_{0}^{t}\left[\theta_{a}(u(\tau))-1\right] R_{\tau}[u] d \tau .
$$

The set of Eqs. (A8) and (A10) solves the problem of calculating the characteristic functional of the stochastic pulse process (A1).

Furthermore, we consider some particular cases. First of all, we try to find from Eqs. (A8) and (A10) an explicit expression for the characteristic functional of noise with the number of pulses distributed according to the Poissonian statistics, which corresponds to the exponential probability distribution of interspike intervals $w(\zeta)=\lambda e^{-\lambda \zeta}(\zeta \geqslant 0)$. Here $1 / \lambda$ is the mean time interval between neighboring pulses. Substituting this distribution in the integral equation (A8), we get

$$
R_{t}[u]=\lambda e^{-\lambda t}+\lambda \int_{0}^{t} e^{-\lambda(t-\tau)} \theta_{a}(u(\tau)) R_{\tau}[u] d \tau .
$$

One can transform this integral equation in differential form by differentiating both sides of Eq. (A11) with respect to $t$. As a result, we arrive at

$$
\dot{R}_{t}[u]=\lambda\left[\theta_{a}(u(t))-1\right] R_{t}[u] .
$$

Comparing Eq. (A12) with Eq. (A9), we have

$$
\dot{R}_{t}[u]=\lambda \dot{\Theta}_{t}[u] .
$$

By integrating this equation with the initial condition $R_{0}[u]=$ $\lambda$, obtained from Eq. (A11), we find

$$
R_{t}[u]=\lambda \Theta_{t}[u] .
$$

After substituting Eq. (A13) into Eq. (A9) and performing the integration, we obtain

$$
\Theta_{t}[u]=\exp \left\{\lambda \int_{0}^{t}\left[\theta_{a}(u(\tau))-1\right] d \tau\right\} .
$$

Equation (A14) coincides with that obtained in Ref. [48] for the Poissonian white noise [see the general formula for the characteristic functional of the non-Gaussian white noise and the kernel function in Eqs. (8) and (25) of Ref. [48]].

Now we calculate the characteristic function of the integral of the pulse noise (A1), i.e., the characteristic function of the CTRW process $\alpha(t)$,

$$
\Phi_{t}(u)=\left\langle e^{i u \alpha(t)}\right\rangle=\left\langle\exp \left\{i u \int_{0}^{t} \xi(\tau) d \tau\right\}\right\rangle .
$$

Putting $u(t)=u=$ const in Eq. (A8), we have

$$
R_{t}(u)=w(t)+\theta_{a}(u)\left[w(t) \otimes R_{t}(u)\right],
$$

where $w(t) \otimes R_{t}(u)$ denotes the convolution of two functions. We solve Eq. (A16) by Laplace transform,

$$
\tilde{R}_{s}(u)=\frac{\tilde{w}(s)}{1-\theta_{a}(u) \tilde{w}(s)},
$$

where $\tilde{w}(s)$ and $\tilde{R}_{s}(u)$ are the Laplace transforms of the functions $w(t)$ and $R_{t}(u)$, respectively. Applying the Laplace transform to Eq. (A10), we obtain

$$
\tilde{\Phi}_{s}(u)=\frac{1+\left[\theta_{a}(u)-1\right] \tilde{R}_{s}(u)}{s} .
$$

After substituting Eq. (A17) in this equation, we get

$$
\tilde{\Phi}_{s}(u)=\frac{1-\tilde{w}(s)}{s\left[1-\theta_{a}(u) \tilde{w}(s)\right]} .
$$

Equation (A18) is the Laplace transform of the characteristic function (A15) and was previously obtained in Ref. [47].

For the random pulse train (1) with fixed amplitudes $a_{k}=$ $f$, we have

$$
\tilde{\Phi}_{s}(u)=\frac{1-\tilde{w}(s)}{s\left[1-\tilde{w}(s) e^{i u f}\right]} .
$$

By power-series expansion of Eq. (A19) in the variable $u$, we obtain

$\tilde{\Phi}_{s}(u)=\frac{1}{s}+\frac{i u f \tilde{w}(s)}{s[1-\tilde{w}(s)]}-\frac{u^{2} f^{2} \tilde{w}(s)[1+\tilde{w}(s)]}{2 s[1-\tilde{w}(s)]^{2}}+\cdots$.

From Eq. (A20), one can easily find the Laplace transform of the first two moments of the CTRW process $\alpha(t)$,

$\bar{\alpha}(s)=\frac{f \tilde{w}(s)}{s[1-\tilde{w}(s)]}, \quad \overline{\alpha^{2}}(s)=\frac{f^{2} \tilde{w}(s)[1+\tilde{w}(s)]}{s[1-\tilde{w}(s)]^{2}}$.

Equations (20) and (21) can be obtained from Eq. (A21) in the limit of large $t$.
[1] See the special section on Complex Systems, Science 284, 79 (1999).

[2] O. N. Bjornstad and B. T. Grenfell, Science 293, 638 (2001); see also the special section on Ecology Through Time, Sci- ence 293, 623 (2001); W. Ebeling and B. Spagnolo, Fluct. Noise Lett. 5, L159 (2005); A. Caruso, M. E. Gargano, D. Valenti, A. Fiasconaro, and B. Spagnolo, ibid. 5, L349 (2005). 
[3] A. Provata, I. M. Sokolov, and B. Spagnolo, Eur. Phys. J. B 65 , 307 (2008); see also the topical issue on Ecological Complex Systems, Eur. Phys. J. B 65, 307 (2008); P. Romanczuk, I. D. Couzin, and L. Schimansky-Geier, Phys. Rev. Lett. 102, 010602 (2009).

[4] W. J. Blake, M. Kaern, C. R. Cantor, and J. J. Collins, Nature (London) 422, 633 (2003).

[5] E. M. Ozbudak, M. Thattai, I. Kurtser, A. D. Grossman, and A. van Oudenaarden, Nat. Genet. 1, 69 (2002).

[6] P. Turchin, L. Oksanen, P. Ekerholm, T. Oksanen, and H. Henttonen, Nature (London) 405, 562 (2000).

[7] B. Spagnolo, A. Fiasconaro, and D. Valenti, Fluct. Noise Lett. 3, L177 (2003).

[8] D. Valenti, A. Fiasconaro, and B. Spagnolo, Physica A 331, 477 (2004); B. Spagnolo, M. Cirone, A. La Barbera, and F. de Pasquale, J. Phys. Condens. Matter 14, 2247 (2002); A. La Barbera and B. Spagnolo, Physica A 314, 120 (2002); B. Spagnolo and A. La Barbera, ibid. 315, 114 (2002).

[9] B. Spagnolo, D. Valenti, and A. Fiasconaro, Math. Biosci. Eng. 1, 185 (2004).

[10] H. C. Tuckwell and E. Le Corfec, J. Theor. Biol. 195, 451 (1998).

[11] O. Chichigina, D. Valenti, and B. Spagnolo, Fluct. Noise Lett. 5, L243 (2005).

[12] B. T. Grenfell, K. Wilson, B. F. Finkenstädt, T. N. Coulson, S. Murray, S. D. Albon, J. M. Pemberton, T. H. Clutton-Brock, and M. J. Crawley, Nature (London) 394, 674 (1998).

[13] O. A. Chichigina, Eur. Phys. J. B 65, 347 (2008).

[14] A. Giuffrida, D. Valenti, G. Ziino, B. Spagnolo, and A. Panebianco, Eur. Food Res. Technol. 228, 767 (2009);

E. Korobkova, T. Emonet, J. M. Vilar, T. S. Shimizu, and P. Cluzel, Nature (London) 428, 574 (2004).

[15] N. Pizzolato, D. Persano Adorno, D. Valenti, and B. Spagnolo, Theor. Biosci. 130, doi:10.1007/s12064-011-0127-y (2011); N. Pizzolato, D. Valenti, D. Persano Adorno, and B. Spagnolo, Cent. Eur. J. Phys. 7, 541 (2009); I. Roeder, M. Horn, I. Glauche, A. Hochhaus, M. C. Mueller, and M. Loeffler, Nat. Med. 12, 1181 (2006).

[16] P. Barrera, S. Ciuchi, and B. Spagnolo, J. Phys. A 26, L559 (1993); S. Ciuchi, F. de Pasquale, and B. Spagnolo, Phys. Rev. E 47, 3915 (1993); ibid. 54, 706 (1996); M. B. Elowitz, A. J. Levine, E. D. Siggia, and P. S. Swain, Science 297, 1183 (2002); J. M. Raser and E. K. OShea, ibid. 304, 1811 (2004); J. Paulsson, Nature (London) 427, 415 (2004); L. Cai, N. Friedman, and X. S. Xie, ibid. 440, 358 (2006); A. A. Dubkov and B. Spagnolo, Eur. Phys. J. B 65, 361 (2008).

[17] J. R. Newman, S. Ghaemmaghami, J. Ihmels, D. K. Breslow, M. Noble, J. L. DeRisi, and J. S. Weissman, Nature (London) 441, 840 (2006); A. Bar-Even, J. Paulsson, N. Maheshri, M. Carmi, E. O'Shea, Y. Pilpel, and N. Barkai, Nat. Genet. 38, 636 (2006); J. M. Raser and E. K. O’Shea, Science 309, 5743 (2005); D. Valenti, L. Schimansky-Geier, X. Sailer, and B. Spagnolo, Eur. Phys. J. B 50, 199 (2006); D. Valenti, L. Tranchina, C. Cosentino, M. Brai, A. Caruso, and B. Spagnolo, Ecol. Model. 213, 449 (2008); A. La Cognata, D. Valenti, A. A. Dubkov, and B. Spagnolo, Phys. Rev. E 82, 011121 (2010).

[18] A. S. Mikhailov and A. Yu. Loskutov, Foundations of Synergetics II: Chaos and Noise, 2nd revised and enlarged edition, Springer Series in Synergetics (Springer, Berlin, 1996).
[19] M. A. Muñoz, F. Colaiori, and C. Castellano, Phys. Rev. E 72, 056102 (2005); A. Manor and N. M. Shnerb, Phys. Rev. Lett. 103, 030601 (2009).

[20] L. Arnold, Stochastic Differential Equations: Theory and Applications (Wiley, New York, 1974); A. Brissaud and U. Frisch, J. Math. Phys. 15, 524 (1974).

[21] R. Z. Khas'minskii, Stochastic Stability of Differential Equations (Sijthoff and Noordhoff, Alphen, 1981).

[22] N. G. van Kampen, Phys. Rep. 24, 171 (1976).

[23] A. Schenzle and H. Brand, Phys. Rev. A 20, 1628 (1979).

[24] K. Lindenberg, V. Seshadri, and B. J. West, Phys. Rev. A 22, 2171 (1980); Physica A 105, 445 (1981).

[25] G. N. Bochkov and A. A. Dubkov, Radiophys. Quantum Electron. 24, 844 (1981).

[26] J. Łuczka, T. Czernik, and P. Hänggi, Phys. Rev. E 56, 3968 (1997).

[27] A. Porporato and P. D’Odorico, Phys. Rev. Lett. 92, 110601 (2004).

[28] Y. Wu and W. Q. Zhu, Phys. Rev. E 77, 041911 (2008); Phys. Lett. A 372, 623 (2008).

[29] I. M. Sokolov and V. V. Belik, Physica A 330, 46 (2003).

[30] G. Aquino, M. Bologna, P. Grigolini, and B. J. West, Phys. Rev. E 70, 036105 (2004).

[31] P. Allegrini, F. Barbi, P. Grigolini, and P. Paradisi, Chaos Solitons Fractals 34, 11 (2007).

[32] F. Barbi, M. Bologna, and P. Grigolini, Phys. Rev. Lett. 95, 220601 (2005).

[33] I. M. Sokolov, Phys. Rev. E 73, 067102 (2006).

[34] O. C. Akin, P. Paradisi, and P. Grigolini, Physica A 371, 157 (2006).

[35] O. C. Akin, P. Paradisi, and P. Grigolini, J. Stat. Mech. (2009) P01013.

[36] S. Bianco, P. Grigolini, and P. Paradisi, Chem. Phys. Lett. 438, 336 (2007).

[37] G. Izzo and A. Vecchio, J. Comput. Appl. Math. 210, 210 (2007); S. A. H. Geritz and É. Kisdi, J. Theor. Biol. 228, 261 (2004); R. E. Mickens, Appl. Numer. Math. 45, 309 (2003); A. Ramani, A. S. Carstea, R. Willox, and B. Grammaticos, Physica A 333, 278 (2004).

[38] D. R. Cox, Renewal Theory (Chapman and Hall, New York, 1967).

[39] C. Godreche and J. M. Luck, J. Stat. Phys. 104, 489 (2001).

[40] R. Stratonovich, Topics in the Theory of Random Noise. I (Gordon and Breach, New York, 1963).

[41] Ya. B. Zel'dovich, S. A. Molchanov, A. A. Ruzmaikin, and D. D. Sokolov, Sov. Phys. Usp. 30, 353 (1987).

[42] L. M. Riccardi and F. Esposito, Biol. Cybern. 3, 148 (1966).

[43] E. Jakeman and J. H. Jefferson, Opt. Acta 33, 557 (1986).

[44] G. Faraci and A. R. Pennisi, Phys. Rev. A 33, 583 (1986).

[45] A. Clauset, C. R. Shalizi, and M. E. J. Newman, SIAM Rev. 51, 661 (2009); M. Newman, Nature (London) 405, 412 (2000).

[46] A. Manor and N. M. Shnerb, Phys. Rev. Lett. 103, 030601 (2009); Y.-P. Jeon and B. J. McCoy, Phys. Rev. E 72, 037104 (2005).

[47] E. W. Montroll and G. H. Weiss, J. Math. Phys. 6, 167 (1965).

[48] A. A. Dubkov and B. Spagnolo, Fluct. Noise Lett. 5, L267 (2005). 